\title{
Does cooking with vegetable oils increase the risk of chronic diseases?: a systematic review
}

\author{
Carmen Sayon-Orea ${ }^{1}$, Silvia Carlos ${ }^{1,2}$ and Miguel A. Martínez-Gonzalez ${ }^{1,3 *}$ \\ ${ }^{1}$ Department of Preventive Medicine and Public Health, Facultad de Medicina-Clinica, Universidad de Navarra, \\ Ed. Investigación, C/Irunlarrea 1, 31008 Pamplona, Navarra, Spain \\ ${ }^{2}$ Institute for Culture and Society (ICS), Education of Affectivity and Human Sexuality, University of Navarra, \\ Pamplona, Spain \\ ${ }^{3}$ CIBERobn Physiopathology of Obesity and Nutrition, Institute of Health Carlos III (ISCIII), Madrid, Spain
}

(Submitted 4 March 2014 - Final revision received 23 July 2014 - Accepted 1 August 2014)

\begin{abstract}
Overweight/obesity, CVD and type 2 diabetes are strongly associated with nutritional habits. High consumption of fried foods might increase the risk of these disorders. However, it is not clear whether the use of vegetables oils for cooking increases the risk of chronic diseases. We systematically searched for published studies that assessed the association between vegetable oil consumption including fried food consumption and the risk of overweight/obesity or weight gain, T2DM or the metabolic syndrome, and CVD or hypertension in the following databases: PubMed; Web of Science; Google Scholar. Keywords such as 'fried food' or 'vegetable oil' or 'frying' or 'frying oils' or 'dietary fats' and 'weight gain' or 'overweight' or 'obesity' or 'CHD' or 'CVD' or 'type 2 diabetes' or 'metabolic syndrome' were used in the primary search. Additional published reports were obtained through other sources. A total of twenty-three publications were included based on specific selection criteria. Based on the results of the studies included in the present systematic review, we conclude that (1) the myth that frying foods is generally associated with a higher risk of CVD is not supported by the available evidence; (2) virgin olive oil significantly reduces the risk of CVD clinical events, based on the results of a large randomised trial that included as part of the intervention the recommendation to use high amounts of virgin olive oil, also for frying foods; and (3) high consumption of fried foods is probably related to a higher risk of weight gain, though the type of oil may perhaps modify this association.
\end{abstract}

Key words: Fried foods: Olive oil: Obesity: Hypertension: Metabolic syndrome: Type 2 diabetes mellitus: CVD

Some chronic diseases such as overweight/obesity, CVD and type 2 diabetes mellitus (T2DM) are closely associated with lifestyle factors and nutritional habits ${ }^{(1)}$. In the last few decades, epidemiological evidence has helped to clarify the specific role of diet and its components in the prevention or elevation of the risk of these non-communicable diseases. The role that some dietary components, such as vegetable oils, may play in the determination of the risk of these conditions has been identified by observational studies and intervention trials. Since the pioneering Seven Countries

Abbreviations: HR, hazard ratios; MetS, metabolic syndrome; PREDIMED, Prevención con Dieta Mediterránea; SUN, Seguimiento Universidad de Navarra; T2DM, type 2 diabetes mellitus.

*Corresponding author: Professor Dr M. A. Martinez-Gonzalez, fax +34948 455649, email mamartinez@unav.es

Tublication of these papers was supported by unrestricted educational grants from Federación Española de Sociedades de Nutrición, Alimentación y Dietética (FESNAD), International Nut and Dried Fruit Council (INC), International Union of Nutritional Sciences (IUNS), Fundación Iberoamericana de Nutrición (FINUT), Centro de Investigación Biomédica en Red de la Fisiopatología de la Obesidad y Nutrición (CIBERobn) and Centro Interuniversitario di Ricerca sulle Culture Alimentari Mediterranee (Ciiscam). The papers included in this supplement were invited by the Guest Editors and have undergone the standard journal formal review process. They may be cited. The Guest Editors declare that Salas-Salvadó is a nonpaid member of the World Forum for Nutrition Research and Dissemination of the International Nut and Dried Fruit Council. Angel Gil is President of the Fundación Iberomericana de Nutrición, which is a non-paid honorary position. Lluis Serra-Majem is the President of the Scientific Committee of the Mediterranean Diet Foundation and Scientific Director of the CIISCAM (Centro Interuniversitario di Ricerca sulle Culture Alimentari Mediterranee), Universita La Sapienza di Roma which are both non-paid, honorary positions. Goretti Guasch is the Executive Director and Member of the Executive Committee of the International Nut and Dried Fruit Council, which is a paid position. Mònica Bulló declares no conflict of interest. 
Study, dietary SFA have been considered to be directly associated with higher serum cholesterol concentrations and with a higher risk of CVD. In this context, high SFA intake was considered to be the primary determinant of CVD risk in Western countries. Moreover, recommending reduction of the intake of all types of fats was considered to be a practical approach to reduce saturated fat intake, and this recommendation was included in almost all the dietary guidelines during the last two to three decades ${ }^{(2)}$. The high energy density and high palatability of high-fat foods were feared to exert potentially adverse effects on body weight and cardiovascular health. In addition, Ornish et $a l^{(3)}$ reported a regression of coronary atherosclerosis in a study carried out in forty-eight patients with angiographically documented CHD, in which the intervention group (twenty-eight patients) followed a low-fat diet with $<10 \%$ of total energy as fat. This small study contributed to the building up of the myth that all types of fats, including vegetable oils, are detrimental for cardiovascular health. This belief opened the door to the recommendation of lowfat diets for the prevention of CVD. However, the results obtained in large randomised trials using low-fat diets as a paradigm of a healthy diet to prevent the development of CVD or obesity have been disappointing ${ }^{(4-6)}$. A reduction in fat intake is usually compensated by a proportional increase in carbohydrate intake. In the current cultural context, most carbohydrates are highly refined and may even be more favourable for the development of obesity, T2DM and CVD than fats. The isoenergetic comparison of carbohydrate $v$. SFA rendered both equally detrimental for cardiovascular health $^{(7)}$, and when the comparison was made between $n-6$ PUFA (present in seed oils) and SFA, the intake of PUFA was found to be significantly associated with a reduced risk of CHD. In these circumstances, an alternative dietary paradigm that is different from the low-fat diet and can be more useful for developing and implementing programmes aimed at achieving prolonged weight loss and improving cardiovascular health is the traditional Mediterranean diet. This dietary pattern is rich in fat from vegetable oils (especially virgin olive oil) and includes an abundance of minimally processed plant foods (vegetables, fruits, whole grains and legumes), low consumption of meat (especially red and processed meats), moderate consumption of fish and wine (which is usually consumed with meals) and frugal meals. The high fat content of the traditional Mediterranean diet makes it more palatable and therefore more acceptable and easily sustainable in the long term. It is likely that a higher intake of vegetable oils may contribute to a greater palatability and consequently sustainability.

Vegetable oils are produced from oilseeds (i.e. sunflower), legumes (i.e. soyabean), nuts (i.e. almond) or the flesh of some fruits (i.e. olives). They are mainly composed of TAG and therefore serve as sources of fat. As all sources of fat, vegetable oils, contain different kinds of fatty acids, their compositions vary widely, but typically one type of fatty acid will predominate over the others; for example, the major fatty acid in olive oil is oleic acid, a MUFA, and the predominant fatty acid in sunflower oil is linoleic acid, a PUFA $^{(8)}$. Concerning vegetable oils and health, besides the predominant fatty acid in each specific oil, another issue that has to be taken into consideration is the culinary purpose, because oil has different effects on health depending on the form it is eaten (used for dressing, for cooking or for frying).

Therefore, we conducted a systematic review of the existing evidence regarding the association of vegetable oil consumption including fried food consumption with the risk of overweight/obesity or weight gain, T2DM or the metabolic syndrome (MetS), and CVD or hypertension.

\section{Methods}

A systematic search for published studies that assessed the association between vegetable oil consumption including fried food consumption and the risk of overweight/obesity or weight gain, T2DM or the MetS, and CVD and hypertension was performed in the following databases: PubMed; Web of Science; Google Scholar. Keywords such as 'fried food' or 'frying' or 'frying oils' or 'vegetable oil' or 'dietary fats' and 'weight gain' or 'overweight' or 'obesity' or 'CHD' or 'CVD' or 'type 2 diabetes' or 'metabolic syndrome' were used in the primary search. Additional published reports were obtained through other sources. The language of publication was restricted to English and Spanish. Studies that had a case-control, cohort or randomised controlled trial study design; those that considered vegetable oil consumption or fried food consumption as the exposure of interest; and those that investigated weight gain, overweight, obesity, T2DM, the MetS, CVD or hypertension as the outcome were included in the present systematic review. Reviews, editorials, comments, letters, meeting abstracts, short communications, protocols, non-human studies, studies that did not specifically consider vegetable oil or fried food consumption as exposure, and studies that did not consider weight gain, overweight, obesity, T2DM, the MetS or CVD as the outcome were excluded.

\section{Data extraction}

The following information was extracted from each included study: author and year of publication; study design; dietary intake assessment method; number of FFQ items; exposure (type of vegetable oil or fried food consumption) and categorisation; follow-up period; number of participants included and characteristics of the included participants such as age, sex and country; the outcome (weight gain, overweight, obesity, T2DM, the MetS, and CVD or hypertension); number of cases and controls in case-control studies, number of events in cohort and randomised controlled trial studies; the covariates included in the adjusted models and $\beta$-regression coefficients, OR and hazard ratios (HR) were also extracted.

\section{Results}

\section{Literature search}

The results of the literature search are shown in Fig. 1. In total, 3549 records were identified in PubMed, 388 records in the 


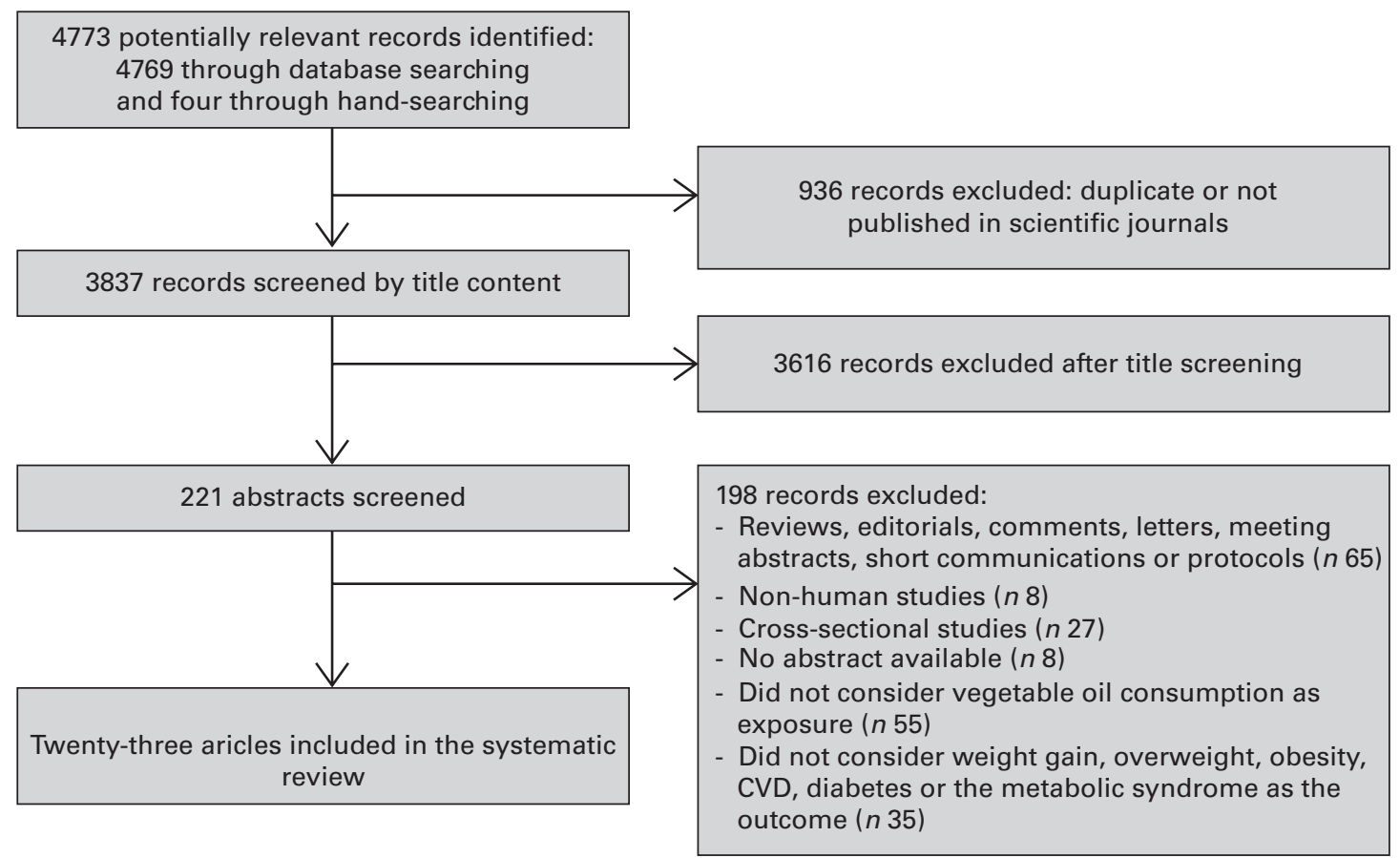

Fig. 1. Flow diagram depicting the identification and selection processes of relevant studies that investigated the association between vegetable oil or fried food consumption and the risk of overweight/obesity or weight gain, T2DM or the metabolic syndrome, and CVD or hypertension.

Web of Science and 832 in Google Scholar until April 2014; four additional records were identified through other sources. After exclusion of duplicate records and records that were not published in scientific journals ( $n$ 936), 3837 records were screened. In the first instance, records were screened only by title content and 3616 records were excluded. The abstracts of the remaining 221 records were read and records that did not meet the inclusion criteria were excluded, leaving a total of twenty-three articles for inclusion in the systematic review.

\section{Study characteristics}

The information extracted from the twenty-three studies included in the present systematic review is summarised in Tables $1-3$. Studies that investigated weight gain or the risk of overweight or obesity as the outcome were conducted in the $\mathrm{USA}^{(9,10)}$ and Spain ${ }^{(11-13)}$; studies that assessed the incidence of T2DM or the MetS were also conducted in the $\mathrm{USA}^{(14,15)}$ and Spain ${ }^{(16-19)}$; and studies that investigated the risk of CVD and hypertension were conducted in the $\mathrm{USA}^{(20)}$, Spain ${ }^{(21-25)}$ India $^{(26)}$, Costa Rica ${ }^{(27)}$, Norway ${ }^{(28)}$, Greece $^{(29)}$, Italy ${ }^{(30)}$ and France ${ }^{(31)}$ (Table 2).

Vegetable oil and fried food consumption was measured using FFQ (in the majority of the studies), dietary history questionnaire $^{(22,23)}$ or $24 \mathrm{~h}$ dietary recall ${ }^{(31)}$. Dietary intake information was updated in seven studies ${ }^{(9,10,14-16,18,25)}$ by repeated measurements of dietary intake using the FFQ during the follow-up period (Table 1).

A summary of the results discussed in the review is shown in Fig. 2.

\section{Association between vegetable oil or fried food consumption and weight gain or the risk of overweight/obesity}

A total of five cohort studies ${ }^{(9-13)}$ that investigated the association between vegetable oil or fried food consumption and weight gain or the risk overweight/obesity were included in the present systematic review. Fried food consumption was found to be positively associated with the risk of becoming overweight/obese, as well as with a greater weight gain $^{(9,10,13)}$. On the other hand, high consumption of olive oil was found to be not associated with a higher risk $^{(11)}$, and a negative association was even reported by some studies ${ }^{(12)}$.

The most relevant results in this context were from the study of Mozaffarian et $a l{ }^{(9)}$, which using data of three separate large US cohorts investigated the relationship between multiple dietary changes including fried food consumption and long-term weight gain. The following three cohorts were included in this study: (1) the Nurses' Health Study (NHS): a prospective cohort study of 121701 female registered nurses enrolled in 1976; (2) the NHS-II: a prospective cohort study of 116686 younger female registered nurses enrolled in 1989; (3) the Health Professionals Follow-up Study (HPFUS): a prospective cohort study of 51529 male health professionals enrolled in 1986. All cohort participants were followed up using mailed questionnaires every 2 years. In total, 50422 women from the NHS, 47898 women from the NHS-II and 22557 men from the HPFUS were found to be eligible, thereby 120877 US women and men, all free of obesity and chronic diseases at baseline and who had complete data on weight and lifestyle habits, were included 


\section{N British Journal of Nutrition}

Table 1. Study design characteristics of the twenty-three epidemiological studies that investigated the association between vegetable oil or fried food consumption (FFC) and weight change, incident overweight/obesity, type 2 diabetes, the metabolic syndrome, hypertension or CVD

\begin{tabular}{|c|c|c|c|c|c|c|c|}
\hline Author and year & $\begin{array}{l}\text { Dietary intake } \\
\text { assessment } \\
\text { method }\end{array}$ & FFQ items & $\begin{array}{l}\text { Type of } \\
\text { vegetable oil } \\
\text { consumption or FFC }\end{array}$ & Categorisation & $\begin{array}{l}\text { Validation } \\
\text { of nutrients }\end{array}$ & $\begin{array}{l}\text { Multiple } \\
\text { assessments of } \\
\text { dietary intake }\end{array}$ & $\begin{array}{l}\text { Average } \\
\text { duration of } \\
\text { follow-up (years) }\end{array}$ \\
\hline \multicolumn{8}{|c|}{ BMI or weight change (continuous variable) } \\
\hline $\begin{array}{l}\text { Taveras } \\
\quad \text { et al. } \\
{ }^{(10)} \\
(2005)\end{array}$ & FFQ & 132 & FFC & $\begin{array}{l}\text { Never or }<1 \\
\quad \text { v. } 4-7 \text { times/week }\end{array}$ & Yes & Yes & 3 \\
\hline $\begin{array}{l}\text { Mozaffarian } \\
\text { et al. }{ }^{(9)}(2011)\end{array}$ & FFQ & NR & $\begin{array}{l}\text { FFC, potato } \\
\text { chips and French fries }\end{array}$ & Increased dietary intake & Yes & Yes & $\begin{array}{c}\mathrm{NHS}=20 ; \mathrm{NHS}-\mathrm{II}=12 ; \\
\text { Health Professionals } \\
\text { Follow-up Study }=20\end{array}$ \\
\hline \multicolumn{8}{|l|}{ Overweight/obesity } \\
\hline $\begin{array}{l}\text { Bes-Rastrollo } \\
\text { et al. }{ }^{(11)}(2006)\end{array}$ & FFQ & 136 & Olive oil & Quintiles & Yes & Baseline & $2 \cdot 4$ \\
\hline $\begin{array}{l}\text { Haro-Mora } \\
\quad \text { et al. }{ }^{(12)}(2011)\end{array}$ & $\begin{array}{l}\text { Frequency of } \\
\text { consumption of } \\
\text { main foods in Spain }\end{array}$ & NR & $\begin{array}{l}\text { Olive oil and } \\
\text { mixture or other } \\
\text { types of oils } \\
\text { (usually sunflower oil) }\end{array}$ & Olive oil $v$. others & No & Baseline & 1 \\
\hline $\begin{array}{l}\text { Sayon-Orea } \\
\quad \text { et al. }{ }^{(13)}(2013)\end{array}$ & FFQ & 136 & FFC & $\begin{array}{l}<2,2-4, \text { or } \\
\quad>4 \text { times/week }\end{array}$ & Yes & Baseline & $6 \cdot 1$ \\
\hline \multicolumn{8}{|l|}{ T2DM } \\
\hline $\begin{array}{l}\text { Halton } \\
\quad \text { et al. }{ }^{(14)}(2006)\end{array}$ & FFQ & 61 & French fries & Quintiles & Yes & Yes & 20 \\
\hline $\begin{array}{l}\text { Salas-Salvado } \\
\quad \text { et al. }{ }^{(16)}(2011)\end{array}$ & FFQ & 137 & EVOO & $\begin{array}{l}\text { Control group, MedDiet } \\
\text { supplemented with } \\
\text { EVOO, and MedDiet } \\
\text { supplemented with nuts }\end{array}$ & Yes & Yes & 4 \\
\hline $\begin{array}{l}\text { Mari-Sanchis } \\
\text { et al. }{ }^{(17)}(2011)\end{array}$ & FFQ & 136 & Olive oil & Quintiles & Yes & Baseline & 5.7 \\
\hline $\begin{array}{l}\text { Salas-Salvado } \\
\text { et al. }{ }^{(18)}(2014)\end{array}$ & FFQ & 137 & EVOO & $\begin{array}{l}\text { Control group, MedDiet } \\
\text { supplemented with EVOO, } \\
\text { and MedDiet } \\
\text { supplemented with nuts }\end{array}$ & Yes & Yes & $4 \cdot 1$ \\
\hline \multicolumn{8}{|l|}{ Metabolic syndrome } \\
\hline $\begin{array}{l}\text { Lutsey } \\
\quad \text { et al. } \\
{ }^{(15)}(2008)\end{array}$ & FFQ & 66 & FFC & Tertiles & NR & Yes & 9 \\
\hline $\begin{array}{l}\text { Sayon-Orea } \\
\quad \text { et al. }{ }^{(19)}(2014)\end{array}$ & FFQ & 136 & FFC & $\begin{array}{l}0-2,>2-4, \text { or } \\
>4 \text { times } / \text { week }\end{array}$ & Yes & Baseline & $8 \cdot 3$ \\
\hline \multicolumn{8}{|l|}{ CVD } \\
\hline $\begin{array}{l}\text { Mozaffarian } \\
\quad \text { et al. }{ }^{(20)}(2003)\end{array}$ & FFQ & NR & Fried fish & $\begin{array}{l}<1 \text { time/month, } \\
1-3 \text { times/month, } \\
1 \text { time/week, } 2 \text { times/week, } \\
\text { or } \geq 3 \text { times/week }\end{array}$ & Yes & Baseline & $9 \cdot 3$ \\
\hline $\begin{array}{l}\text { Rastogi } \\
\quad \text { et al. }{ }^{(26)}(2004)\end{array}$ & FFQ & 141 & $\begin{array}{l}\text { Cooking or frying with } \\
\text { sunflower oil (ref.), ghee, } \\
\text { vanaspati, mustard oil, } \\
\text { peanut oil or safflower oil }\end{array}$ & $\begin{array}{l}\text { Type of oil used for } \\
\text { cooking or frying }\end{array}$ & Yes & Baseline & NA \\
\hline $\begin{array}{l}\text { Kabagambe } \\
\text { et al. }{ }^{(27)}(2005)\end{array}$ & FFQ & NR & $\begin{array}{l}\text { Palm oil } v \text {. soyabean } \\
\text { oil and others (including } \\
\text { sunflower, maize, olive } \\
\text { and rapeseed oils) }\end{array}$ & Type of oil used for cooking & Yes & Baseline & NA \\
\hline $\begin{array}{l}\text { Lockheart } \\
\quad \text { et al. }{ }^{(28)}(2007)\end{array}$ & FFQ & 190 & $\begin{array}{l}\text { Non-hydrogenated } \\
\text { vegetable oil }\end{array}$ & Tertiles & Yes & Baseline & NA \\
\hline $\begin{array}{l}\text { Kontogianni } \\
\quad \text { et al. }{ }^{(29)}(2007)\end{array}$ & FFQ & NR & Olive oil and others & $\begin{array}{l}\text { No use of olive oil } v \text {. } \\
\text { exclusive use of olive } \\
\text { oil and olive oil plus others }\end{array}$ & NR & Baseline & NA \\
\hline
\end{tabular}




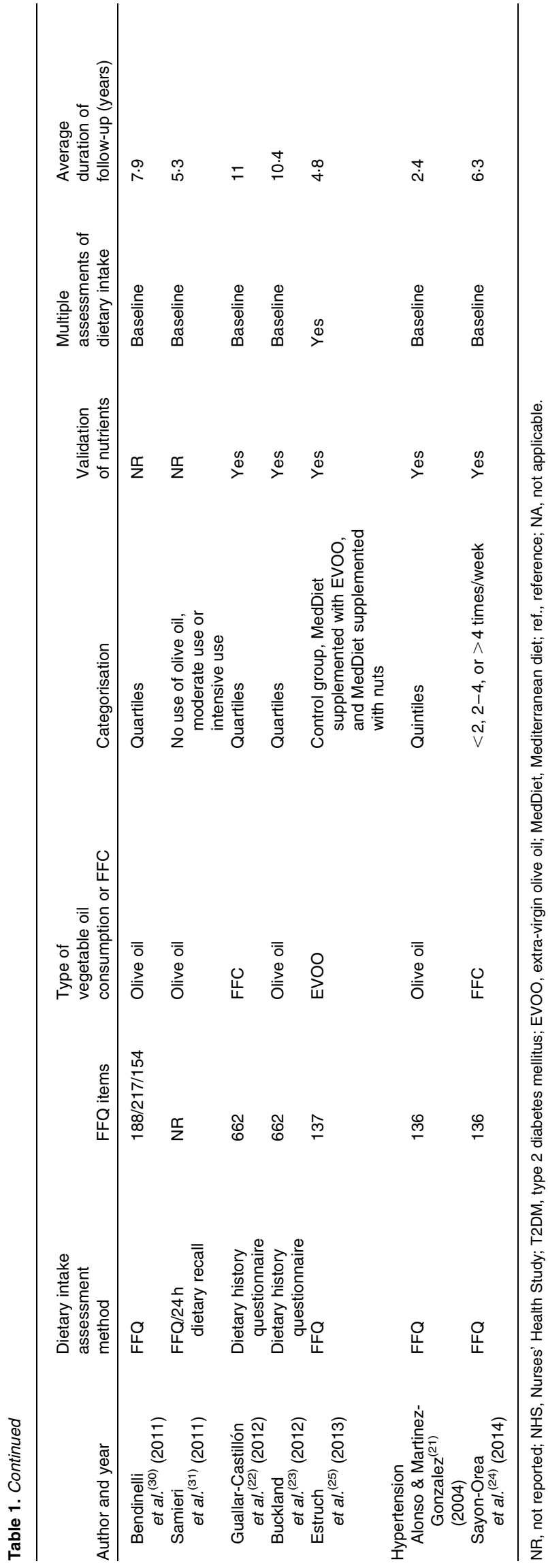

in the study. The relationship between changes in lifestyle factors and weight change was evaluated every 4 years. The dietary factors with the largest positive association with weight change/serving per $\mathrm{d}$ were increases in the consumption of potato chips and French fries (weight change after 4 years was $1.69,95 \% \mathrm{CI} 1.30,2 \cdot 09 \mathrm{lb}$, and $3.35,95 \% \mathrm{CI}$ $2 \cdot 29,4.42 \mathrm{lb}$, respectively). Fried food consumption at home and away from home was also found to be significantly associated with weight gain $(0.39 \mathrm{lb}, 95 \%$ CI $0 \cdot 22,0.51$ and $0.28 \mathrm{lb}$, $95 \%$ CI $0 \cdot 08,0 \cdot 48$ respectively).

The 'Seguimiento Universidad de Navarra' (SUN) study assessed the association between fried food consumption and weight gain in a sample of 9850 participants with a mean age of 38.1 (SD 11.4) years who were followed up for a median time period of $6 \cdot 1$ years ${ }^{(13)}$. Fried food consumption was found to be not significantly associated with a higher weight gain ( $\beta$-regression coefficient $=+46$, 95\% CI, $-17,+109 \mathrm{~g} ; P$ for trend $=0 \cdot 230)$ on comparing the highest $(>4$ times/week) $v$. the lowest $(<2$ times/week) category of consumption. The risk of overweight/obesity was also analysed in this study in a sample of 6821 participants initially free of overweight/obesity at baseline, and during the same follow-up period, 1068 incident cases were identified, and higher consumption of fried foods was found to be associated with a significantly higher risk of becoming overweight/obese (OR 1.37, $95 \mathrm{CI} \% 1.08,1.73 ; P$ for trend=0.021) when comparing the highest $(>4$ times/week) $v$. the lowest $(<2$ times/week) category of consumption. In this study, analyses stratified by the type of oil used for frying were also conducted, in which it was found that those who used olive oil for frying tended to gain less weight and had a lower risk of becoming overweight/obese; however, none of these associations was found to be statistically significant. The results of this study supported the hypothesis that a high frequency of fried food consumption was associated with the risk of becoming overweight/obese among young adults in a Spanish cohort with a high educational level.

The possibility that frying with olive oil might be healthier and could be considered as a preferable substitute for frying with other oils deserves further assessments ${ }^{(32)}$.

Some authors have shown that the impact of fried food consumption on body weight does not depend on the place where fried foods are prepared (home or away from home $)^{(9)}$, with fried foods being prepared both at home and away from home being found to increase the risk of overweight/obesity. Therefore, it is assumed that the frying process (pan-frying or deep-frying, number of frying sessions, duration and temperature of frying, etc.) is not a determinant of body weight increase. However, most of the literature regarding the health effects of frying foods on overweight/obesity does not take into account specific aspects of the frying process, and it is well known that the frying technology can affect both the quality and the amount of fats consumed ${ }^{(33)}$. Among the oils used for cooking, olive oil is less prone to oxidation ${ }^{(34)}$ and it maintains its healthbeneficial properties, even after being cooked due to its thermal stability. It has even been shown that the antioxidants 
Table 2. Characteristics of the twenty-three epidemiological studies that investigated the association between vegetable oil or fried food consumption and weight change, incident overweight/obesity, type 2 diabetes, the metabolic syndrome, hypertension or CVD

(Mean values and standard deviations)

\begin{tabular}{|c|c|c|c|c|c|c|}
\hline Author and year & No. of participants & Age (years) & Country & Time of data acquisition & Sex (\% male) & Outcome \\
\hline \multicolumn{7}{|l|}{ BMI or weight change (continuous variable) } \\
\hline Taveras et al. ${ }^{(10)}(2005)$ & 14355 & Range: $9-14$ & USA & $1996-9$ & $46 \cdot 1$ & Annual change in BMI \\
\hline Mozaffarian et al. $^{(9)}(2011)$ & 120877 & $\begin{array}{l}\mathrm{NHS}=52.2(\operatorname{SD} 7.2) \\
\mathrm{NHS}-1 \mathrm{I}=37.5(\operatorname{SD} 4.1) \\
\text { HPFUS }=50.8(\operatorname{SD} 7.5)\end{array}$ & USA & $1988-2006$ & $18 \cdot 7$ & Weight change \\
\hline \multicolumn{7}{|l|}{ Overweight/obestiy } \\
\hline Bes-Rastrollo et al. ${ }^{(11)}(2006)$ & 5356 & Range: $20-90$ & Spain & 1999-2004 & 41.5 & Incidence of overweight or obesity \\
\hline Haro-Mora et al. ${ }^{(12)}$ (2011) & 92 & Range: $13-166$ months & Spain & NR & 80.4 & Risk of increased BMI \\
\hline Sayon-Orea et al. ${ }^{(13)}(2013)$ & 6821 & $38.1(\mathrm{SD} 11.4)$ & Spain & $1999-2010$ & $46 \cdot 1$ & Risk of overweight or obesity \\
\hline \multicolumn{7}{|l|}{ T2DM } \\
\hline Halton et al. ${ }^{(14)}(2006)$ & 84555 & Range: $34-59$ & USA & $1980-2000$ & 0 & Type 2 diabetes \\
\hline Salas-Salvado et al. ${ }^{(16)}(2011)$ & 418 & $67 \cdot 3$ & Spain & $2003-8$ & 41.6 & Type 2 diabetes \\
\hline Mari-Sanchis et al. ${ }^{(17)}(2011)$ & 10491 & $38.9(\mathrm{SD} 11.4)$ & Spain & $1999-2009$ & $47 \cdot 2$ & Type 2 diabetes \\
\hline Salas-Salvado et al. ${ }^{(18)}(2014)$ & 3541 & Range: $55-80$ & Spain & $2003-2010$ & 38.0 & Type 2 diabetes \\
\hline \multicolumn{7}{|l|}{ Metabolic syndrome } \\
\hline Lutsey et al. ${ }^{(15)}(2008)$ & 9514 & $53 \cdot 6$ & USA & $1987-1998$ & $44 \cdot 1$ & Metabolic syndrome \\
\hline Sayon-Orea et al. ${ }^{(19)}(2014)$ & 8289 & $35.9($ SD 10.4) & Spain & $1999-2012$ & 33.9 & Metabolic syndrome \\
\hline \multicolumn{7}{|l|}{ CVD } \\
\hline Mozaffarian et al. ${ }^{(20)}$ (2003) & 3910 & $\geq 65$ & USA & $1989-2000$ & $39 \cdot 0$ & IHD \\
\hline Rastogi et al. ${ }^{(26)}(2004)$ & 1050 & Range: $21-74$ & India & $1999-2000$ & 88 & IHD \\
\hline Kabagambe et al. ${ }^{(27)}(2005)$ & 4222 & $\begin{array}{l}\text { Cases = 58.5 (SD 11.0) } \\
\quad \text { controls = 58.2 }(\text { SD 11.3) }\end{array}$ & Costa Rica & $1994-2004$ & 73 & Non-fatal acute myocardial infarction \\
\hline Lockheart et al. (28) $^{(2007)}$ & 211 & $62.5(\mathrm{SD} 7.7)$ & Norway & $1995-7$ & NR & First myocardial infarction \\
\hline Kontogianni et al. ${ }^{(29)}(2007)$ & 1926 & NR & Greece & NR & 82.5 & Non-fatal acute coronary syndrome \\
\hline Bendinelli et al. ${ }^{(30)}(2011)$ & 29689 & Range: $35-74$ & Italy & 1993-8 & 0 & $\mathrm{CHD}$ \\
\hline Samieri et al. ${ }^{(31)}(2011)$ & 7625 & $73 \cdot 8(\mathrm{SD} 5 \cdot 3)$ & France & $1999-2005$ & $37 \cdot 7$ & Stroke \\
\hline Guallar-Castillón et al. ${ }^{(22)}$ (2012) & 40757 & Range: $29-69$ & Spain & $1992-2004$ & 37.7 & CHD \\
\hline Buckland et al. ${ }^{(23)}(2012)$ & 40142 & Range: $29-69$ & Spain & 1992-2004 & 38 & $\mathrm{CHD}$ \\
\hline Estruch et al. ${ }^{(25)}(2013)$ & 7447 & Range: $55-80$ & Spain & $2003-2010$ & 43.0 & $\mathrm{CHD}$ \\
\hline \multicolumn{7}{|l|}{ Hypertension } \\
\hline Alonso \& Martinez-Gonzalez ${ }^{(21)}(2004)$ & 5573 & $>20$ & Spain & $2000-$ & $39 \cdot 3$ & Hypertension \\
\hline Sayon-Orea et al. ${ }^{(24)}(2014)$ & 13679 & $36.5(S D 10.8)$ & Spain & $1999-2012$ & 37.0 & Hypertension \\
\hline
\end{tabular}

HPFUS, Health Professionals Follow-up Study; NHS, Nurses' Health Study; NR, not reported, T2DM, type 2 diabetes mellitus. 
Table 3. Results for association between vegetable oil or fried food consumption and weight change, incident overweight/obesity, type 2 diabetes, the metabolic syndrome, hypertension or CVD reported by the twenty-three epidemiological studies

(Odds ratios, hazard ratios or $\beta$-coefficients and $95 \%$ confidence intervals)

\begin{tabular}{|c|c|c|c|c|c|c|c|}
\hline Author and year & Design & $\begin{array}{l}\text { No. of } \\
\text { cases }\end{array}$ & $\begin{array}{l}\text { No. of } \\
\text { controls }\end{array}$ & $\begin{array}{l}\text { No. of } \\
\text { events }\end{array}$ & Covariates in the adjusted model & $\beta$-Coefficient or OR/HR & $95 \% \mathrm{Cl}$ \\
\hline \multicolumn{8}{|c|}{ BMI or weight change (continuous variable) } \\
\hline $\begin{array}{l}\text { Taveras } \\
\quad \text { et al. }{ }^{(10)}(2005)\end{array}$ & Cohort & NA & NA & NA & $\begin{array}{l}\text { Age, race/ethnicity, baseline and follow-up menstrual status (girls), } \\
\text { Tanner stage, baseline height, annual change in height, } \\
\text { previous BMI Z-score, physical activity and sex }\end{array}$ & $\beta$-Coefficient $=0.21 \mathrm{~kg}$ & $0.03,0.39$ \\
\hline $\begin{array}{l}\text { Mozaffarian } \\
\text { et al. }{ }^{(9)}(2011)\end{array}$ & Cohort & NA & NA & NA & $\begin{array}{l}\text { Age, baseline BMI, sleep duration, changes in physical activity, } \\
\text { alcohol consumption, time spent watching television, } \\
\text { smoking status and dietary factors }\end{array}$ & $\begin{array}{l}\text { Potato chips: } 1.69 \mathrm{lb} \text {; } \\
\text { French fries: } 3.35 \mathrm{lb} \text {; } \\
\text { fried foods: } 0.39 \mathrm{lb}\end{array}$ & $\begin{array}{l}1.30,2 \cdot 09 \\
2 \cdot 29,4 \cdot 42 \\
0 \cdot 22,0.51\end{array}$ \\
\hline \multicolumn{8}{|l|}{ Overweight/obesity } \\
\hline $\begin{array}{l}\text { Bes-Rastrollo } \\
\text { et al. }^{(11)}(2006)\end{array}$ & Cohort & NA & NA & 405 & $\begin{array}{l}\text { Age, sex, total energy intake, fibre intake, vegetable consumption, } \\
\text { leisure-time physical activity, smoking status, snacking } \\
\text { between meals, time spent watching television and baseline BMI }\end{array}$ & $\mathrm{OR}=1 \cdot 11$ & $0 \cdot 76,1 \cdot 61$ \\
\hline $\begin{array}{l}\text { Haro-Mora } \\
\text { et al. } .^{(12)}(2011)\end{array}$ & Cohort & NA & NA & NR & Age, baseline BMI and physical activity & $\mathrm{OR}=0.19$ & $0.06,0.61$ \\
\hline $\begin{array}{l}\text { Sayon-Orea } \\
\quad \text { et al. }{ }^{(13)}(2013)\end{array}$ & Cohort & NA & NA & 1068 & $\begin{array}{l}\text { Age, sex, time spent sitting down ( } \mathrm{h} / \text { week), baseline BMI, } \\
\text { smoking status, physical activity, nut intake, sugar-sweetened } \\
\text { beverage (SSB) consumption, fibre intake, snacking, total } \\
\text { energy intake, family history of obesity and fast food consumption }\end{array}$ & $\mathrm{OR}=1.37$ & $1 \cdot 08,1 \cdot 73$ \\
\hline \multicolumn{8}{|c|}{ - } \\
\hline $\begin{array}{l}\text { Halton } \\
\quad \text { et al. }^{(14)}(2006)\end{array}$ & Cohort & NA & NA & 4496 & $\begin{array}{l}\text { Age, BMI, family history of diabetes, smoking status, } \\
\text { postmenopausal hormone use, physical activity, } \\
\text { total energy intake, cereal fibre intake, trans-fat } \\
\text { intake and polyunsaturated fat:saturated fat }\end{array}$ & $\mathrm{RR}=1 \cdot 16$ & $1 \cdot 05,1 \cdot 29$ \\
\hline $\begin{array}{l}\text { Salas-Salvado } \\
\text { et al. } .^{(16)}(2011)\end{array}$ & PREDIMED RCT & NA & NA & 54 & $\begin{array}{l}\text { Sex, age, baseline energy intake, BMI, waist circumference, } \\
\text { physical activity, smoking status, fasting serum glucose } \\
\text { concentrations, use of lipid-lowering drugs, MedDiet score } \\
\text { and weight changes during the study }\end{array}$ & $\mathrm{HR}=0.49$ & $0.25,0.97$ \\
\hline $\begin{array}{l}\text { Mari-Sanchis } \\
\quad \text { et al. } .^{(17)}(2011)\end{array}$ & Cohort & NA & NA & 42 & $\begin{array}{l}\text { Age, sex, BMI, physical activity, family history of diabetes, } \\
\text { gestational diabetes, hypercholesterolaemia, hypertension } \\
\text { and total energy intake }\end{array}$ & $\mathrm{OR}=1.04$ & $0.43,2.55$ \\
\hline $\begin{array}{l}\text { Salas-Salvado } \\
\text { et al. } .^{(18)}(2014)\end{array}$ & $\mathrm{RCT}$ & NA & NA & 273 & $\begin{array}{l}\text { Age, sex, BMI, smoking status, fasting glucose concentrations, } \\
\text { prevalence of dyslipidaemia, hypertension, total energy intake, } \\
\text { adherence to the MedDiet, physical activity, } \\
\text { educational level and alcohol consumption }\end{array}$ & $\mathrm{HR}=0.60$ & $0.43,0.85$ \\
\hline \multicolumn{8}{|l|}{ Metabolic Syndrome } \\
\hline $\begin{array}{l}\text { Lutsey } \\
\qquad \text { et al. }{ }^{(15)}(2008)\end{array}$ & Cohort & NA & NA & 3782 & Age, sex, race, educational level, centre and total energy intake & $\mathrm{HR}=1.25$ & $1 \cdot 14,1 \cdot 37$ \\
\hline $\begin{array}{l}\text { Sayon-Orea } \\
\text { et al. }{ }^{(19)}(2014)\end{array}$ & Cohort & NA & NA & 420 & $\begin{array}{l}\text { Age, sex, baseline BMI, time spent sitting down, } \\
\text { smoking status, physical activity, sugared soft drink intake, } \\
\text { fibre intake, fast food consumption, snacking between meals, } \\
\text { categories of MedDiet, total energy intake, and carbohydrate, } \\
\text { fat and } \mathrm{Na} \text { intake }\end{array}$ & $\mathrm{HR}=0.93$ & $0 \cdot 72,1 \cdot 20$ \\
\hline \multicolumn{8}{|c|}{ 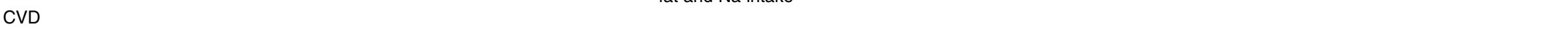 } \\
\hline $\begin{array}{l}\text { Mozaffarian } \\
\text { et al. }^{(20)}(2003)\end{array}$ & Cohort & NA & NA & 247 & $\begin{array}{l}\text { Age, sex, educational level, diabetes, current smoking status, } \\
\text { pack-year of smoking, BMI, systolic blood pressure, } \\
\text { LDL-cholesterol concentrations, HDL-cholesterol concentrations, } \\
\text { TAG concentrations, C-reactive protein concentrations } \\
\text { and saturated fat intake }\end{array}$ & $\mathrm{HR}=1.37$ & $0.48,3.90$ \\
\hline
\end{tabular}


Table 3. Continued

\begin{tabular}{|c|c|c|c|c|c|c|c|}
\hline Author and year & Design & $\begin{array}{l}\text { No. of } \\
\text { cases }\end{array}$ & $\begin{array}{l}\text { No. of } \\
\text { controls }\end{array}$ & $\begin{array}{l}\text { No. of } \\
\text { events }\end{array}$ & Covariates in the adjusted model & $\beta$-Coefficient or OR/HR & $95 \% \mathrm{Cl}$ \\
\hline $\begin{array}{l}\text { Rastogi } \\
\text { et al. }{ }^{(26)}(2004)\end{array}$ & Case-control & 350 & 700 & NA & $\begin{array}{l}\text { Age, sex, hospital, smoking status, BMI, waist:hip ratio, } \\
\text { physical activity, hypertension, diabetes, hypercholesterolaemia, } \\
\text { family history of IHD, alcohol consumption, educational level, } \\
\text { household income, religion, cereal intake and } \\
\text { green leafy vegetable intake }\end{array}$ & $\begin{array}{l}\text { Cooking in sunflower } \\
\text { v. mustard oil: } R R=0.46 ; \\
\text { frying in sunflower } \\
\text { v. mustard oil: } R R=0.25\end{array}$ & $\begin{array}{l}0.22,0.95 \\
0.11,0.57\end{array}$ \\
\hline $\begin{array}{l}\text { Kabagambe } \\
\text { et al. }{ }^{(27)}(2005)\end{array}$ & Case-control & 2111 & 2111 & NA & $\begin{array}{l}\text { Age, sex, area of residence, smoking status, alcohol consumption, } \\
\text { diabetes, hypertension, abdominal obesity, physical activity, } \\
\text { income, total energy intake and fibre intake }\end{array}$ & $\begin{array}{l}\text { Other oils } v \text {. palm oil: } \\
\qquad \mathrm{OR}=1 \cdot 23\end{array}$ & $0.99,1.52$ \\
\hline $\begin{array}{l}\text { Lockheart } \\
\quad \text { et al. }{ }^{(28)}(2007)\end{array}$ & Case-control & 106 & 105 & NA & $\begin{array}{l}\text { Age, marital status educational level, family history of } \\
\text { heart disease, smoking status and energy intake }\end{array}$ & $\mathrm{OR}=0.53$ & $0.24,1.18$ \\
\hline $\begin{array}{l}\text { Kontogianni } \\
\text { et al. }{ }^{(29)}(2007)\end{array}$ & Case-control & 848 & 1078 & NA & $\begin{array}{l}\text { Age, sex, BMI, smoking status, physical activity, } \\
\text { educational level, family history of CHD, hypertension, } \\
\text { hypercholesterolaemia and diabetes }\end{array}$ & $\begin{array}{l}\text { No use } v \text {. exclusive use of } \\
\text { olive oil: } \mathrm{OR}=0.53\end{array}$ & $0.34,0.71$ \\
\hline $\begin{array}{l}\text { Bendinelli } \\
\text { et al. } .^{(30)}(2011)\end{array}$ & Cohort & NA & NA & 144 & $\begin{array}{l}\text { Educational level, smoking status, alcohol consumption, } \\
\text { height, weight, waist circumference, daily non-alcohol } \\
\text { energy intake, hypertension, menopausal status, total } \\
\text { physical activity and meat consumption }\end{array}$ & $\mathrm{HR}=0.56$ & $0.31,0.99$ \\
\hline $\begin{array}{l}\text { Samieri } \\
\quad \text { et al. }{ }^{(31)}(2011)\end{array}$ & Cohort & NA & NA & 148 & $\begin{array}{l}\text { Age, sex, educational level, centre, consumption of fish, } \\
\text { meat, pulses, raw vegetables, cereals, } n \text {-3-rich oils, butter, } \\
\text { goose, and duck fat, alcohol consumption physical activity, } \\
\text { risk factors for stroke, BMI, triacylglycerolaemia } \\
\text { and hypercholesterolaemia }\end{array}$ & $\mathrm{HR}=0.59$ & $0.37,0.94$ \\
\hline $\begin{array}{l}\text { Guallar-Castillón } \\
\text { et al. } \text { (22) }^{(2012)}\end{array}$ & Cohort & NA & NA & 606 & $\begin{array}{l}\text { Age, sex, centre, alcohol consumption, educational level, } \\
\text { smoking status, physical activity, diabetes, hyperlipidaemia, } \\
\text { cancer, oral contraceptive use, menopause, hormone } \\
\text { replacement therapy, consumption of fruits, nuts, } \\
\text { dairy products and non-fried foods, BMI, waist } \\
\text { circumference and hypertension }\end{array}$ & $\mathrm{HR}=1.08$ & $0.82,1.43$ \\
\hline $\begin{array}{l}\text { Buckland } \\
\quad \text { et al. }{ }^{(23)}(2012)\end{array}$ & Cohort & NA & NA & 587 & $\begin{array}{l}\text { Age, sex, centre, alcohol consumption, educational level, } \\
\text { BMI, waist circumference, physical activity, smoking status, } \\
\text { alcohol consumption, energy intake, hyperlipidaemia, } \\
\text { hypertension, diabetes and MedDiet score }\end{array}$ & $\mathrm{HR}=0.85$ & $0.68,1.07$ \\
\hline $\begin{array}{l}\text { Estruch } \\
\quad \text { et al. } \\
\text { (25) }(2013)\end{array}$ & $\mathrm{RCT}$ & NA & NA & 288 & $\begin{array}{l}\text { Sex, age, family history of premature CHD, smoking status, } \\
\text { BMI, waist:height ratio, hypertension at baseline, } \\
\text { dyslipidaemia at baseline and diabetes at baseline }\end{array}$ & $\mathrm{HR}=0.70$ & $0.54,0.92$ \\
\hline \multicolumn{8}{|c|}{ 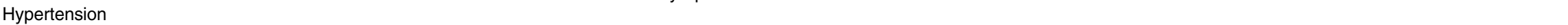 } \\
\hline $\begin{array}{l}\text { Alonso \& Martinez- } \\
\text { Gonzalez } \\
(2004)\end{array}$ & Cohort & NA & NA & 161 & $\begin{array}{l}\text { Age, sex, BMI, leisure-time physical activity, } \\
\text { total energy intake, alcohol consumption, } \\
\text { Na intake and } \mathrm{Ca} \text { intake }\end{array}$ & $\begin{array}{l}\text { OR overall }=0.63 \\
\quad \text { OR women }=0.97 \\
\text { OR men }=0.46\end{array}$ & $\begin{array}{l}0.36,1 \cdot 07 \\
0.40,2 \cdot 36 \\
0.23,0.94\end{array}$ \\
\hline $\begin{array}{l}\text { Sayon-Orea } \\
\quad \text { et al. }{ }^{(24)}(2014)\end{array}$ & Cohort & NA & NA & 1232 & $\begin{array}{l}\text { Sex, age, family history of hypertension, self-reported } \\
\text { hypercholesterolaemia, physical activity, smoking status, } \\
\text { total energy intake, alcohol consumption, energy-adjusted } \\
\mathrm{Na} \text { and K intake, caffeine intake, fibre intake, olive oil intake, } \\
\text { fruit intake, vegetable intake, low-fat and high-fat dairy } \\
\text { product intake, sugar-sweetened beverage intake, fast food } \\
\text { and sweets consumption, and time spent watching television }\end{array}$ & $\mathrm{HR}=1 \cdot 18$ & $1 \cdot 01,1 \cdot 38$ \\
\hline
\end{tabular}

NA, not applicable; NR, not reported; T2DM, type 2 diabetes mellitus; RR, relative risk; RCT, randomised controlled trial; MedDiet, Mediterranean diet; HR, hazard ratio. 


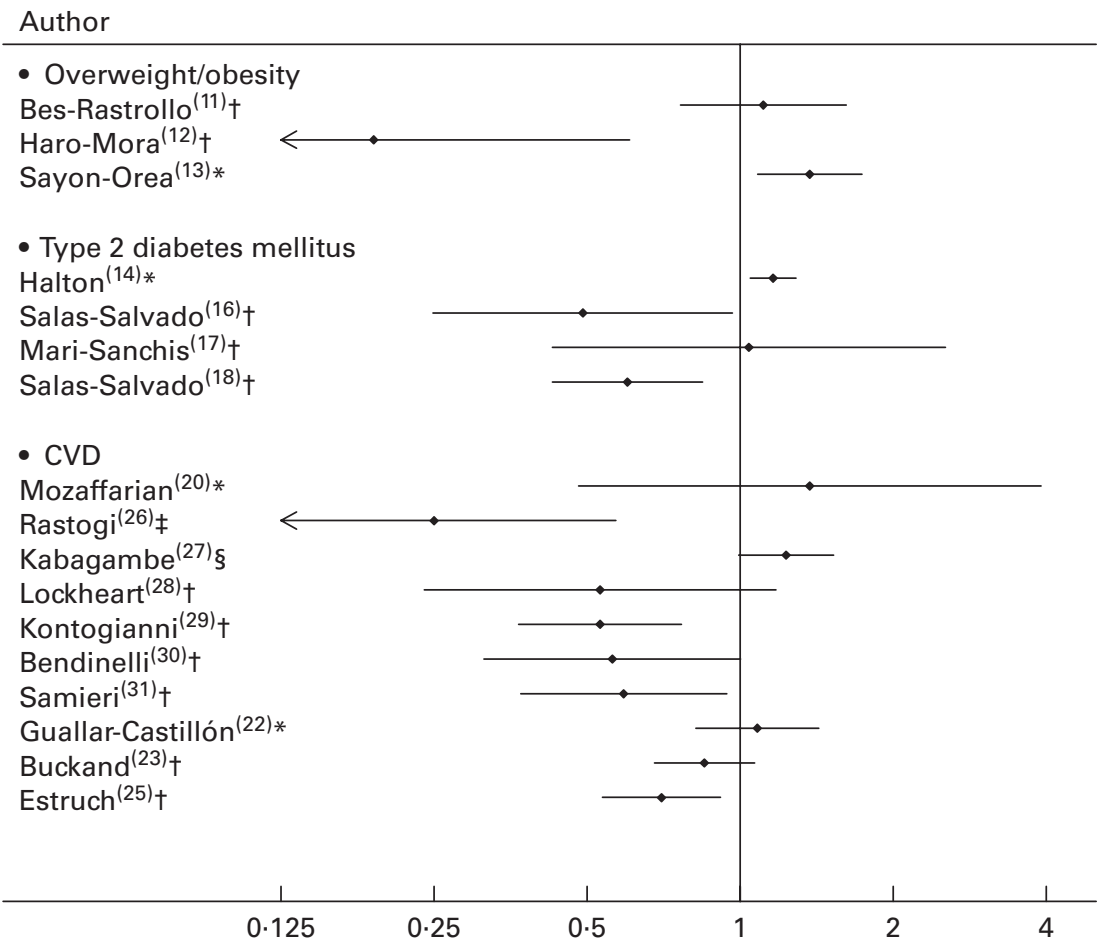

Fig. 2. OR/HR and $95 \% \mathrm{Cl}$ for the fully adjusted model in the studies included in the present systematic review. ${ }^{*}$ Fried food consumption as exposure. $\dagger$ Olive oil consumption as exposure. ‡Sunflower oil consumption as exposure. § Palm oil consumption as exposure.

in frying oils (natural or added) might protect against postprandial oxidative stress in obese individuals ${ }^{(35)}$.

Furthermore, during cooking, molecular interactions occur between oil compounds and the different foods cooked. Oil becomes part of the product ${ }^{(36)}$ and can therefore alter other food components during cooking, being able to increase or decrease the health and protective effects of some cooked foods. An exchange of lipid compounds between the oil used for frying and the food (fish) being fried has been demonstrated, although it depends on the fat content of the raw food. Furthermore, both the type of fish and the type of oil were found to influence the oxidation status of the lipid fraction. Ansorena et ll $^{(33)}$ found that the type of oil used for frying significantly affected the nutritional quality of low-fat fish than that of fatty fish as they absorb more fat. Zotos et al. ${ }^{(37)}$ found frying foods in olive oil to lead to milder changes in the lipid profile compared with frying them in sunflower oil. They found a complete change in the fatty acid profile of anchovy samples, but the beneficial fatty acids EPA and DHA and the $n-3: n-6$ ratio remained at satisfactory levels. They found a high reduction in the concentrations of fish beneficial fatty acids and a tremendous reduction of the $n-3: n-6$ ratio when sunflower oil was used for frying. Frying in olive or sunflower oil was found to reduce cholesterol concentrations and significantly increase squalene (an antioxidant intermediate in cholesterol biosynthesis) concentrations, particularly when using olive oil ${ }^{(37)}$. Naseri et $a l .{ }^{(38)}$ reported a decrease in the food $n-3$ fatty acid content and an increase in the $n-6$ content as a consequence of the migration of fatty acids from fish to the frying oil. FloresAlvarez et al. ${ }^{(36)}$ also showed that the type of fried food influences the oil used for frying: oil degradation was found to be faster for fish nuggets than for French fries, and this degradation may influence the oil content of the specific food type fried. This oil uptake has been shown to differ depending on the frying time or pressure ${ }^{(39)}$. When extravirgin olive oil is used for frying French fries, fish or different vegetables, oil absorption occurs and the food absorbs antioxidant compounds that get enriched with polyphenols.

\section{Association between vegetable oil or fried food consumption and the risk of type 2 diabetes or the metabolic syndrome}

A total of four cohort studies ${ }^{(14,15,17,19)}$ and two studies ${ }^{(16,18)}$ analysing the results of the PREDIMED (Prevención con Dieta Mediterránea) study, a randomised controlled trial, that assessed the association between vegetable oil or fried food consumption and the risk of T2DM or the MetS were included in the present systematic review. Fried food consumption was found to be directly associated with the risk of $\mathrm{T}_{2} \mathrm{DM}^{(14)}$ and the MetS ${ }^{(15)}$. However, the results of the SUN study indicated no association between fried food consumption and the risk of the $\mathrm{MetS}^{(19)}$. The PREDIMED study found an inverse association between olive oil consumption and the risk of $\mathrm{T}_{2} \mathrm{DM}^{(16,18)}$, while the SUN study did not find any association between olive oil consumption and the risk of $\mathrm{T}_{2} \mathrm{DM}^{(17)}$.

The PREDIMED study is a parallel-group, multi-centre, randomised trial that included 7447 participants (men aged 55-80 years; women aged 60-80 years) with no CVD at enrolment, who had either T2DM or at least three of the following major risk factors: smoking; hypertension; elevated 
LDL-cholesterol concentrations; low HDL-cholesterol concentrations; overweight or obesity; a family history of premature $\mathrm{CHD}^{(40)}$. The participants were randomly assigned, at a 1:1:1 ratio, to one of the following three dietary intervention groups: a Mediterranean diet supplemented with extra-virgin olive oil (free provision of 1 litre/week for the participant and his/her family); a Mediterranean diet supplemented with nuts (a free $30 \mathrm{~g} / \mathrm{d}$ provision of walnuts, hazelnuts and almonds); a control low-fat diet. The principal aim of this study was to assess the association between adherence to the Mediterranean diet and cardiovascular risk; however, secondary outcomes such as T2DM were also assessed. As part of the intervention, the participants randomly allocated to the two Mediterranean diet groups were advised (on a quarterly basis) to exclusively use olive oil for culinary purposes, including for frying. Salas-Salvado et al. ${ }^{(16)}$ analysing only one participating centre of those included in the PREDIMED study and excluding participants with T2DM at baseline found that a Mediterranean diet supplemented with olive oil in comparison with the control diet reduced the risk of T2DM (HR 0.49; 95\% CI 0.25, 0.97), and they reported that the possible biological mechanism responsible for this effect might be the anti-inflammatory properties of the virgin olive oil that might be relevant to the reduction of the risk of T2DM. Recently, the PREDIMED investigators have published the results regarding T2DM, but using data from all the centres ${ }^{(18)}$. A total of 3541 patients free of T2DM at baseline were included, and participants in the group of Mediterranean diet supplemented with olive oil were found to exhibit a significantly lower risk of developing new-onset T2DM after the follow-up period (HR 0.60; $95 \%$ CI 0.43, 0.85).

The next outcome that was analysed in the present systematic review was the MetS as an ancillary component to complement the results obtained for T2DM. The MetS is a constellation of metabolic abnormalities that increase the risk of heart disease and $\mathrm{T}_{2} \mathrm{DM}^{(41)}$. These metabolic abnormalities include hypertriacylglycerolaemia, low HDL-cholesterol concentrations, elevated blood pressure, impaired glucose metabolism and central obesity ${ }^{(42)}$. The data of 8289 participants initially free of any MetS criterion were analysed in the SUN study and the participants were followed up for a median time period of 8.3 years. The mean age of the participants was 35.9 (SD 10.4) years. This study concluded that frequent consumption of fried foods was not associated with the incidence of the MetS (HR 0.93; 95\% CI 0.72, 1.20; $P$ for trend $=0.538)$ by comparing the highest $(>4$ times/week) $v$. the lowest $(<2$ times/week) category of consumption. However, two of the five components of the MetS, namely central adiposity and high blood pressure, were found to be positively associated with fried food consumption (HR 1.09; $95 \%$ CI $1 \cdot 00,1 \cdot 18 ; P$ for trend=0.040, and HR $1 \cdot 18 ; 95 \% \mathrm{CI}$ $1 \cdot 04,1 \cdot 34 ; P$ for trend $=0.017$, respectively) when comparing the highest $v$. the lowest category of consumption after multivariable adjustment. In the same study, a higher (but not statistically significant) risk of developing the MetS was observed among participants in the highest category of fried food consumption and who used fats other than olive oil for frying (HR 1.26; 95\% CI $0.71,2 \cdot 23 ; P$ for trend=0.446).
The risk of developing the MetS among those in the highest category of consumption but who used olive oil for frying was lower (HR $0.85 ; 95 \%$ CI $0.63,1.15 ; \quad P$ for trend $=0 \cdot 284)$. Therefore, it might be possible that different types of oils used for frying may have different effects that could explain these findings ${ }^{(19)}$. In this context, as a part of the Pizarra study ${ }^{(32)}$, it was assessed whether insulin resistance was cross-sectionally associated with cooking oils. Samples of cooking oil being used were obtained from the kitchen of 538 subjects, and after administration of $75 \mathrm{~g}$ of glucose, oral glucose tolerance tests were conducted. The results of this study showed that the levels of insulin resistance were significantly lower in subjects who used olive oil for frying than in those who used sunflower or a mixture of several oils.

The possible biological mechanism could be explained by the fact that olive oil can reduce the glycaemic load of a meal, decreasing carbohydrate absorption rate, insulin secretion and lipogenesis. MUFA appear to be powerful stimulators of Glucagon-like peptide-1 (GLP1) secretion after an olive oil-enriched meal, leading to better insulin sensitivity ${ }^{(12)}$.

\section{Association between vegetable oil or fried food consumption and the risk of CVD}

A total of seven cohort studies ${ }^{(20-24,30,31)}$, four case-control studies $^{(26-29)}$ and one randomised controlled trial study ${ }^{(25)}$ that investigated the association between vegetable oil or fried food consumption and the risk of CVD were included in the present systematic review. The most relevant finding in this context was the contribution of the PREDIMED study; this study concluded that a Mediterranean diet supplemented with extra-virgin olive oil led to a relative $30 \%$ risk reduction in the incidence of major cardiovascular events among individuals at high cardiovascular risk. The PREDIMED intervention study recommended the use of olive oil for frying in the two active arms of the trial.

In contrast, in some observational studies, fried food consumption was found to be directly associated with a higher risk of $\mathrm{CVD}^{(20)}$ and hypertension ${ }^{(24)}$, whereas olive oil consumption ${ }^{(23,29-31)}$, use of vegetable oils ${ }^{(28)}$ and mustard oil consumption ${ }^{(26)}$ were found to be inversely associated with the risk of CVD. However, palm oil consumption was reported to be positively associated with the risk of $\mathrm{CVD}^{(27)}$. Olive oil consumption was also reported to be inversely associated with the risk of hypertension ${ }^{(21)}$

The European Prospective Investigation into Cancer and Nutrition (EPIC) is a cohort study of 520000 European men and women that aimed to assess the impact of dietary, lifestyle and genetic factors on the risk of cancer and other chronic conditions. EPIC-Spain is a part of this large European cohort study and comprised 41440 participants.

The association between fried food consumption and the risk of CHD was investigated in the EPIC-Spain. A total of 40757 healthy adults were included in this study. During a median follow-up period of 11 years, 606 incident cases of CHD were identified. The multivariate HR for the fourth (highest) quartile compared with the first (lowest) quartile of fried 
food consumption was $1.08(0.82,1.43 ; P$ for trend=0.74). The results of this study did not vary when stratified by the type of oil used for frying (olive oil/sunflower oil). In this study, only olive oil and sunflower oil were evaluated; therefore, authors admitted that they cannot dismiss that frying with other types of fats may still be harmful ${ }^{(22)}$.

The association between fried food consumption and the risk of hypertension was investigated in the SUN cohort, including data of 13679 participants (5059 men and 8620 women) free of hypertension at baseline. The mean age of the participants was 36.5 (SD $10 \cdot 8$ ) years. During a median follow-up period of 6.3 years, 1232 incident cases of hypertension were identified. The adjusted HR for developing hypertension among participants with a higher frequency of baseline fried food consumption ( $>4$ times/week) was 1.21 (95\% CI 1.04, 1.41) compared with those in the lowest category of consumption $(<2 \text { times/week) ( } P \text { for trend }=0 \cdot 020)^{(24)}$. This result was consistent with that of a cross-sectional study conducted in 538 participants, in which the association between fried food consumption (specifically of reused vegetable oils) and the prevalence of hypertension was evaluated. The results showed that degradation due to the reuse of vegetable oils, especially sunflower oil, was an independent risk factor for hypertension and interestingly the serum concentration of MUFA was found to be negatively associated with this risk ${ }^{(32)}$.

The Cardiovascular Heart Study (CHS) is a populationbased, longitudinal study of CHD and stroke that included participants aged $\geq 65$ years. This study was designed to identify factors related to the onset and course of CHD and stroke, specifically to determine the importance of conventional cardiovascular risk factors in older adults and to identify new risk factors in this age group, especially those that may be protective and modifiable ${ }^{(43)}$. In this context, Mozaffarian et $a l .{ }^{(20)}$ tested the hypothesis that fried fish consumption could be inversely associated with fatal CHD. This study was conducted in 3910 adults free of known CVD at baseline with a mean age of 72 years. Over a mean follow-up period of 9.3 years, 247 fatal CHD cases and 363 incident non-fatal myocardial infarction cases were identified. The consumption of tuna or other broiled or baked fish was found to be associated with a lower risk of total CHD deaths among individuals consuming tuna/other fish $\geq 3$ times/week than among those consuming tuna/other fish $<1$ time/month (HR 0.47; 95\% CI $0.27,0.82 ; P$ for trend=0.002), and contrarily, fried fish consumption was found to be not associated with a lower risk of total CHD deaths (HR 1.37; $95 \%$ CI 0.48, 3.90; $P$ for trend $=0.35$ ) or non-fatal myocardial infarctions (HR 1.93; $95 \%$ CI $0.91,4.08 ; P$ for trend=0.11), but rather to be associated with trends towards a higher risk. Mozaffarian et al. ${ }^{(20)}$ concluded that cardiac benefits of fish consumption vary, according to the type of fish consumed.

The frying process has been reported to increase the hypocholesterolaemic:hypercholesterolaemic fatty acid ratio and reduce the $n-3: n-6$ ratio $^{(44)}$. In addition, Naseri et al. ${ }^{(38)}$ also found that although frying led to a reduction of the $n-3: n-6$ content in olive oil, the reduction was not high. Vegetable oils contain high quantities of $n-6$ PUFA mainly as linoleic acid. The increase in the consumption of linoleic acid-rich oils (e.g. soyabean oil and sunflower oil) is even associated with a decreased $n-3$ long-chain PUFA content in human body tissue. An increased intake of vegetable oils (via excessive use in food processing) characterises high quantities of n-6 PUFA and a deficit in $n-3 \mathrm{PUFA}^{(45)}$. Strobel et al. ${ }^{(45)}$ found that fish processing methods (breading and frying in vegetable oils) had a negative effect, increasing the linoleic acid content and decreasing the n-3:n-6 ratio.

Lipid oxidation is much faster in cooked foods than in fresh foods because cooking accelerates oxidation ${ }^{(39)}$. Olive oil has been reported to confer cardiometabolic health benefits when used for frying. However, in general, frying increases the amounts of cholesterol oxidation products and reduces the activity of paraoxonase, which is an enzyme that inhibits the oxidation of LDL-cholesterol ${ }^{(34)}$. High concentrations of phenolic compounds in olive oil have protective effects against $\mathrm{CVD}^{(46)}$.

\section{Conclusions}

From the results of the studies included in this review, we conclude that (1) the myth that frying foods is generally associated with a higher risk of CVD is not supported by the available evidence; (2) extra-virgin olive oil significantly reduces the risk of CVD clinical events and weight gain, based on the results of a large randomised trial that included as part of the intervention the recommendation to use large amounts of extravirgin olive oil for culinary purposes, also for frying foods; however, the whole Mediterranean dietary pattern plays a more significant role rather than the supplemental extra-virgin olive oil alone; and (3) high consumption of fried foods is probably related to a higher risk of weight gain and hypertension. However, many factors such as the type of oil used and the frying technique (deep-frying or panfrying), frying duration and temperature, and use of new or reused oils for frying have to be taken into account.

The authors' contributions were as follows: MAM-G was responsible for the initial plan and study design, CS-O was responsible for the data collection, data extraction; MAM-G, SC, and CS-O were responsible for the data interpretation, manuscript drafting, critical revision of intellectual content and approval of the final version of the manuscript.

\section{Acknowledgements}

None of the authors has any conflicts of interest to declare.

\section{References}

1. World Health Organization (2003) Diet, Nutrition and the Prevention of Chronic Diseases. Joint WHO/FAO Expert Consultation. WHO Technical Report Series no. 916. Geneva: WHO.

2. Baum SJ, Kris-Etherton PM, Willett WC, et al. (2012) Fatty acids in cardiovascular health and disease: a comprehensive update. J Clin Lipidol 6, 216-234. 
3. Ornish D, Brown SE, Scherwitz LW, et al. (1990) Can lifestyle changes reverse coronary heart disease? The Lifestyle Heart Trial. Lancet 336, 129-133.

4. Howard BV, Van Horn L, Hsia J, et al. (2006) Low-fat dietary pattern and risk of cardiovascular disease: the Women's Health Initiative Randomized Controlled Dietary Modification Trial. JAMA 295, 655-666.

5. Howard BV, Manson JE, Stefanick ML, et al. (2006) Low-fat dietary pattern and weight change over 7 years: the Women's Health Initiative Dietary Modification Trial. JAMA 295, 39-49.

6. Look AHEAD Research Group, Wing RR, Bolin P, et al. (2013) Cardiovascular effects of intensive lifestyle intervention in type 2 diabetes. $N$ Engl J Med 369, 145-154.

7. Jakobsen MU, O'Reilly EJ, Heitmann BL, et al. (2009) Major types of dietary fat and risk of coronary heart disease: a pooled analysis of 11 cohort studies. Am J Clin Nutr 89, $1425-1432$

8. Foster R, Williamson CS \& Lunn J (2009) BRIEFING PAPER: culinary oils and their health effects. Nutr Bull 34, 4-47.

9. Mozaffarian D, Hao T, Rimm E, et al. (2011) Changes in diet and lifestyle and long-term weight gain in women and men. $N$ Engl J Med 364, 2392-2404.

10. Taveras EM, Berkey CS, Rifas-Shiman SL, et al. (2005) Association of consumption of fried food away from home with body mass index and diet quality in older children and adolescents. Pediatrics 116, e518-e524.

11. Bes-Rastrollo M, Sánchez-Villegas A, De la Fuente C, et al. (2006) Olive oil consumption and weight change: the SUN prospective cohort study. Lipids 41, 249-256.

12. Haro-Mora JJ, García-Escobar E, Porras N, et al. (2011) Children whose diet contained olive oil had a lower likelihood of increasing their body mass index $Z$-score over 1 year. Eur J Endocrinol 165, 435-439.

13. Sayon-Orea C, Bes-Rastrollo M, Basterra-Gortari FJ, et al. (2013) Consumption of fried foods and weight gain in a Mediterranean cohort: the SUN project. Nutr Metab Cardiovasc Dis 23, 144-150.

14. Halton TL, Willett WC, Liu S, et al. (2006) Potato and French fry consumption and risk of type 2 diabetes in women. Am J Clin Nutr 83, 284-290.

15. Lutsey PL, Steffen LM \& Stevens J (2008) Dietary intake and the development of the metabolic syndrome: the Atherosclerosis Risk in Communities study. Circulation 117, $754-761$.

16. Salas-Salvado J, Bullo M, Babio N, et al. (2011) Reduction in the incidence of type 2 diabetes with the Mediterranean diet: results of the PREDIMED-Reus nutrition intervention randomized trial. Diabetes Care 34, 14-19.

17. Mari-Sanchis A, Beunza JJ, Bes-Rastrollo M, et al. (2011) Olive oil consumption and incidence of diabetes mellitus, in the Spanish sun cohort. Nutr Hosp 26, 137-143.

18. Salas-Salvado J, Bullo M, Estruch R, et al. (2014) Prevention of diabetes with Mediterranean diets: a subgroup analysis of a randomized trial. Ann Intern Med 160, 1-10.

19. Sayon-Orea C, Martinez-Gonzalez MA, Gea A, et al. (2014) Consumption of fried foods and risk of metabolic syndrome: the SUN cohort study. Clin Nutr 33, 545-549.

20. Mozaffarian D, Lemaitre R, Kuller L, et al. (2003) Cardiac benefits of fish consumption may depend on the type of fish meal consumed: the Cardiovascular Health Study. Circulation 107, 1372-1377.

21. Alonso A \& Martinez-Gonzalez MA (2004) Olive oil consumption and reduced incidence of hypertension: the SUN study. Lipids 39, 1233-1238.
22. Guallar-Castillón P, Rodríguez-Artalejo F, Lopez-Garcia E, et al. (2012) Consumption of fried foods and risk of coronary heart disease: Spanish cohort of the European Prospective Investigation into Cancer and Nutrition study. BMJ 344, e363.

23. Buckland G, Travier N, Barricarte A, et al. (2012) Olive oil intake and $\mathrm{CHD}$ in the European Prospective Investigation into Cancer and Nutrition Spanish cohort. Br J Nutr 108, 2075-2082.

24. Sayon-Orea C, Bes-Rastrollo M, Gea A, et al. (2014) Fried food consumption and the incidence of hypertension in a Spanish cohort: the SUN project. Br J Nutr 112, 984-991.

25. Estruch R, Ros E, Salas-Salvado J, et al. (2013) Primary prevention of cardiovascular disease with a Mediterranean diet. $N$ Engl J Med 368, 1279-1290.

26. Rastogi T, Reddy KS, Vaz M, et al. (2004) Diet and risk of ischemic heart disease in India. Am J Clin Nutr 79, 582-592.

27. Kabagambe EK, Baylin A, Ascherio A, et al. (2005) The type of oil used for cooking is associated with the risk of nonfatal acute myocardial infarction in Costa Rica. J Nutr 135, 2674-2679.

28. Lockheart MS, Steffen LM, Rebnord HM, et al. (2007) Dietary patterns, food groups and myocardial infarction: a case-control study. Br J Nutr 98, 380-387.

29. Kontogianni MD, Panagiotakos DB, Chrysohoou C, et al. (2007) The impact of olive oil consumption pattern on the risk of acute coronary syndromes: The CARDIO2000 casecontrol study. Clin Cardiol 30, 125-129.

30. Bendinelli B, Masala G, Saieva C, et al. (2011) Fruit, vegetables, and olive oil and risk of coronary heart disease in Italian women: the EPICOR Study. Am J Clin Nutr 93, $275-283$.

31. Samieri C, Feart C, Proust-Lima C, et al. (2011) Olive oil consumption, plasma oleic acid, and stroke incidence: the Three-City Study. Neurology 77, 418-425.

32. Soriguer F, Esteva I, Rojo Martinez, et al. (2004) Oleic acid from cooking oils is associated with lower insulin resistance in the general population (Pizarra study). Eur J Endocrinol 150, 33-39.

33. Ansorena D, Guembe A, Mendizabal T, et al. (2010) Effect of fish and oil nature on frying process and nutritional product quality. J Food Sci 75, H62-H67.

34. Cahill LE, Pan A, Chiuve SE, et al. (2014) Fried-food consumption and risk of type 2 diabetes and coronary artery disease: a prospective study in 2 cohorts of US women and men. Am J Clin Nutr 100, 667-675.

35. Perez-Herrera A, Rangel-Zuniga OA, Delgado-Lista J, et al. (2013) The antioxidants in oils heated at frying temperature, whether natural or added, could protect against postprandial oxidative stress in obese people. Food Chem 138, 2250-2259.

36. Flores-Alvarez Mdel C, Molina-Hernandez EF, HernandezRaya JC, et al. (2012) The effect of food type (fish nuggets or French fries) on oil blend degradation during repeated frying. J Food Sci 77, C1136-C1143.

37. Zotos A, Kotaras A \& Mikras E (2013) Effect of baking of sardine (Sardina pilchardus) and frying of anchovy (Engraulis encrasicholus) in olive and sunflower oil on their quality. Food Sci Technol Int 19, 11-23.

38. Naseri M, Abedi E, Mohammadzadeh B, et al. (2013) Effect of frying in different culinary fats on the fatty acid composition of silver carp. Food Sci Nutr 1, 292-297.

39. Pawar DP, Boomathi S, Hathwar SC, et al. (2013) Effect of conventional and pressure frying on lipids and fatty acid 
composition of fried chicken and oil. J Food Sci Technol 50 381-386

40. Martinez-Gonzalez MA, Corella D, Salas-Salvadó J, et al (2012) Cohort profile: design and methods of the PREDIMED study. Int J Epidemiol 41, 377-385.

41. Wilson PWF, D'Agostino R, Parise H, et al. (2005) Metabolic syndrome as a precursor of cardiovascular disease and type 2 diabetes mellitus. Circulation 112, 3066-3072.

42. Grundy S, Brewer HB, Cleeman J, et al. (2004) Definition of metabolic syndrome: report of the National Heart, Lung, and Blood Institute/American Heart Association conference on scientific issues related to definition. Circulation 109, 433-438.
43. Fried LP, Borhani NO, Enright P, et al. (1991) The Cardiovascular Health Study: design and rationale. Ann Epidemiol 1, 263-276.

44. Hosseini H, Mahmoudzadeh M, Rezaei M, et al. (2014) Effect of different cooking methods on minerals, vitamins and nutritional quality indices of kutum roach (Rutilus frisii kutum). Food Chem 148, 86-91.

45. Strobel C, Jahreis G \& Kuhnt K (2012) Survey of $n-3$ and $n-6$ polyunsaturated fatty acids in fish and fish products. Lipids Health Dis 11, 144.

46. Franco MN, Galeano-Diaz T, Lopez O, et al. (2014) Phenolic compounds and antioxidant capacity of virgin olive oil. Food Chem 163, 289-298. 